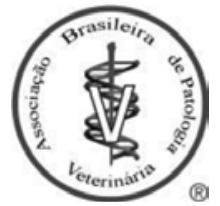

Diagnostic Exercise

From The Latin Comparative Pathology Group*

\title{
Parvovirus enteritis in a raccoon (Procyon lotor)
}

\author{
Contributors:
}

\author{
Omar Gonzales-Viera $^{1 * *}$; Mark Anderson ${ }^{1}$; Patricia Pesavento ${ }^{2}$. \\ ${ }^{1}$ California Animal Health and Food Safety (CAHFS), Davis branch, UC Davis; \\ ${ }^{2}$ Faculty, School of Veterinary Medicine, UC Davis, CA. \\ **Corresponding author: E-mail: gonzalesviera@ucdavis.edu
}

\section{Clinical History:}

Two raccoons died in a pre-release rehabilitation pen in an interval of 1.5 weeks after appearing healthy. The second raccoon, a juvenile female, was submitted for postmortem examination.

\section{Necropsy and Microscopic Findings:}

In the small intestine, the subserosa is markedly hyperemic/congested (Fig. 1) with abundant watery, semi-translucid content and large amounts of tan-white mucus. The intestinal wall is thickened, and the mucosa is smooth and overlaid by thick mucus (Fig. 2). The large intestine contains moderate amounts of tan-yellow, mucoid digesta. Mesenteric lymph nodes are enlarged, the parenchyma is red and mildly protrudes on cut section.

\section{Diagnosis:}

Severe, diffuse, subacute, necrotizing, hemorrhagic, lymphohistiocytic enteritis with rare intranuclear inclusion bodies, coccoid bacteria and coccidia (Fig. 3)

\section{Ancillary tests:}

Direct electron microscopy using feces detected a few 20-22nm diameter viral particles compatible with Parvovirus virions (Fig. 4). Immunohistochemistry with a polyclonal antibody for canine parvovirus immunolabeled viral antigens in the necrotic cells of the crypt necrosis (Fig. 5).

\section{Follow-up questions:}

- Typical microscopic findings of the disease

- Etiological diagnosis

- Most common blood work abnormality.

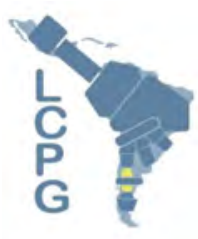

*The Diagnostic Exercises are an initiative of the Latin Comparative Pathology Group (LCPG), the Latin American subdivision of The Davis-Thompson Foundation and published in cooperation with the Brazilian Journal of Veterinary Pathology.

Editor-in-chief for this Diagnostic Exercise: Claudio Barros Associate Editor for this Diagnostic Exercise: Patricia Pesavento

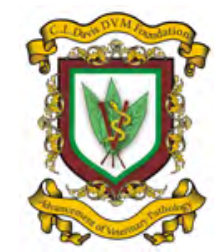




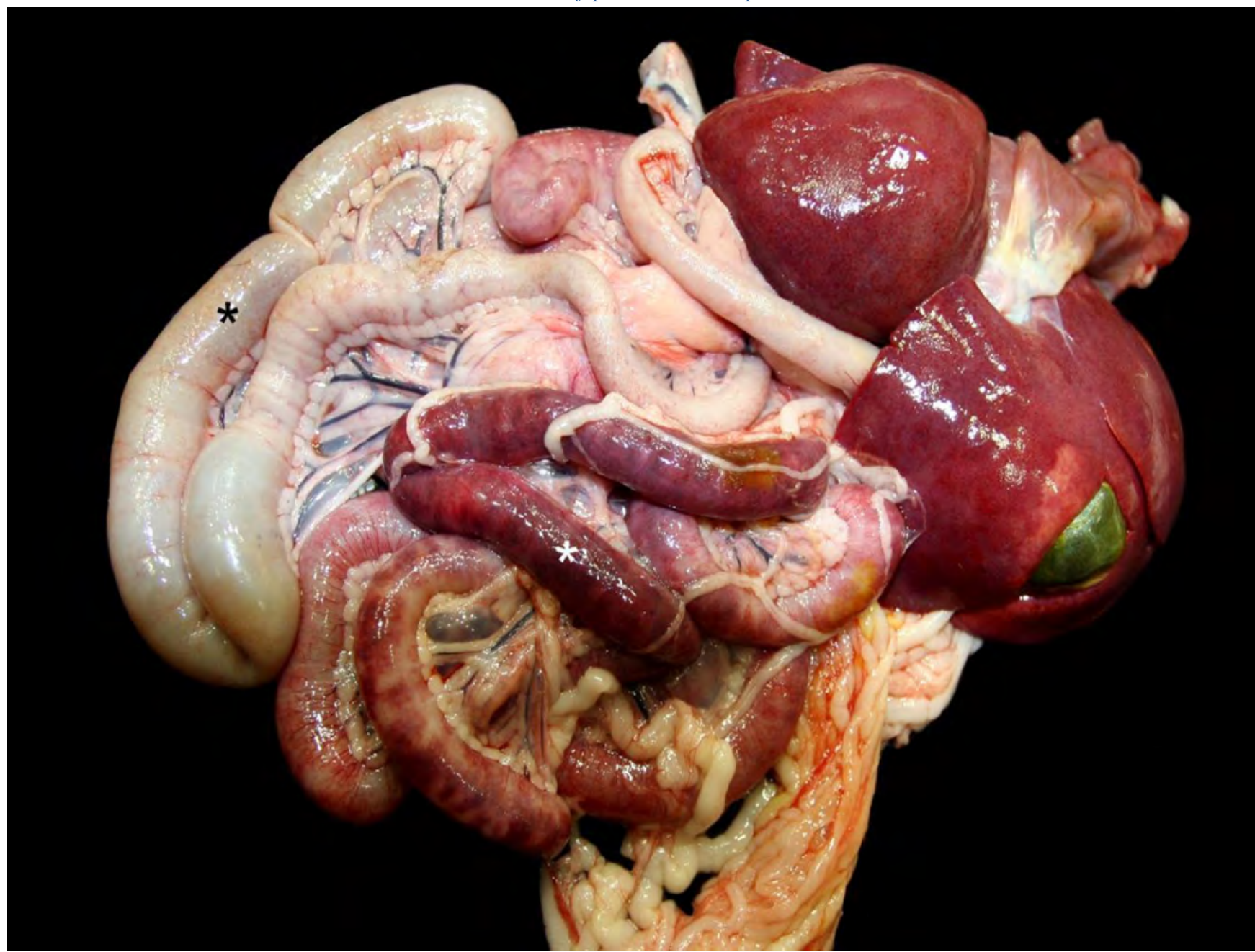

Figure 1. Digestive tract, raccoon, observed the dark-red and slightly sacculated appearance of the small intestine (white asterisk). The large intestine does not depict significant gross findings (black asterisk).

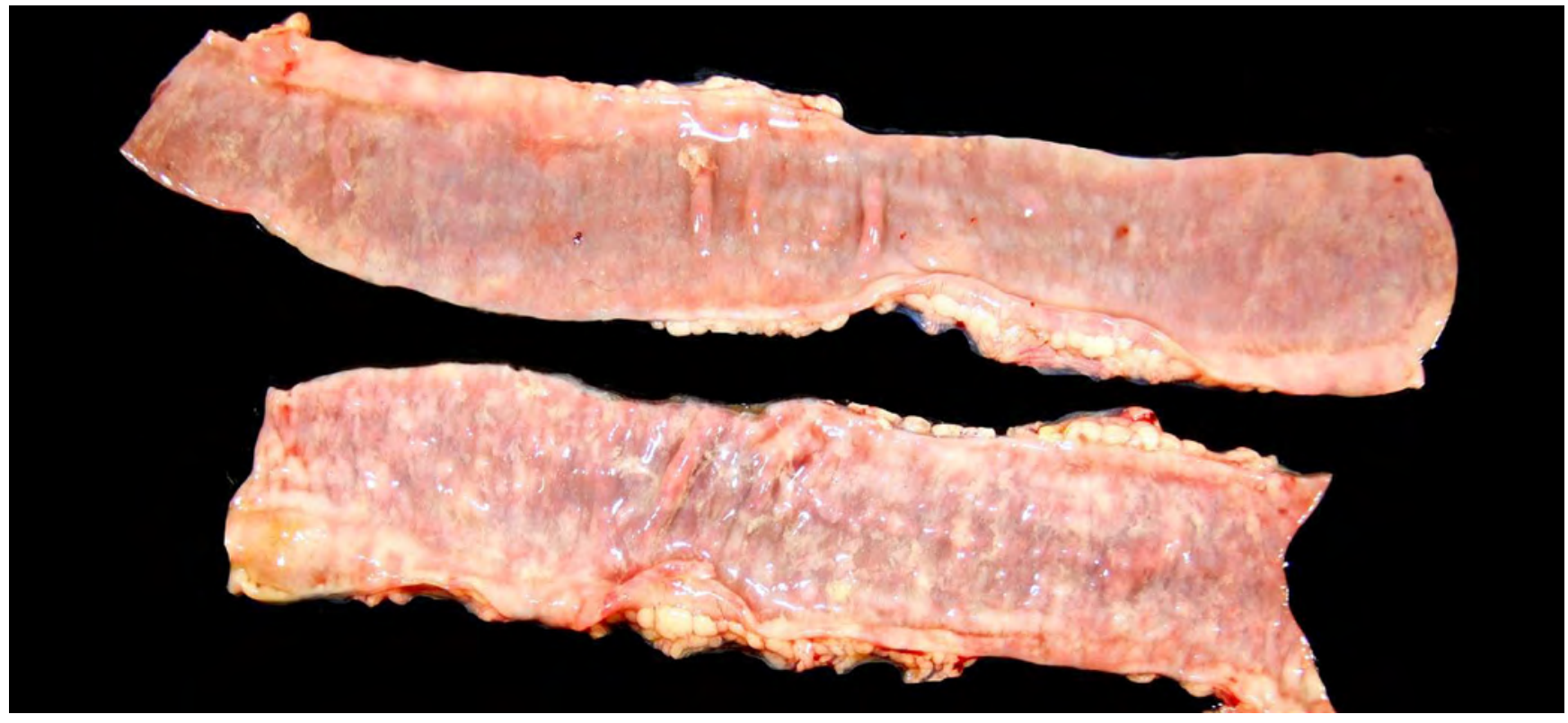

Figure 2. Jejunum, raccoon, two open segments of the jejunum, the mucosa is markedly smooth and covered with tan-white mucus. 


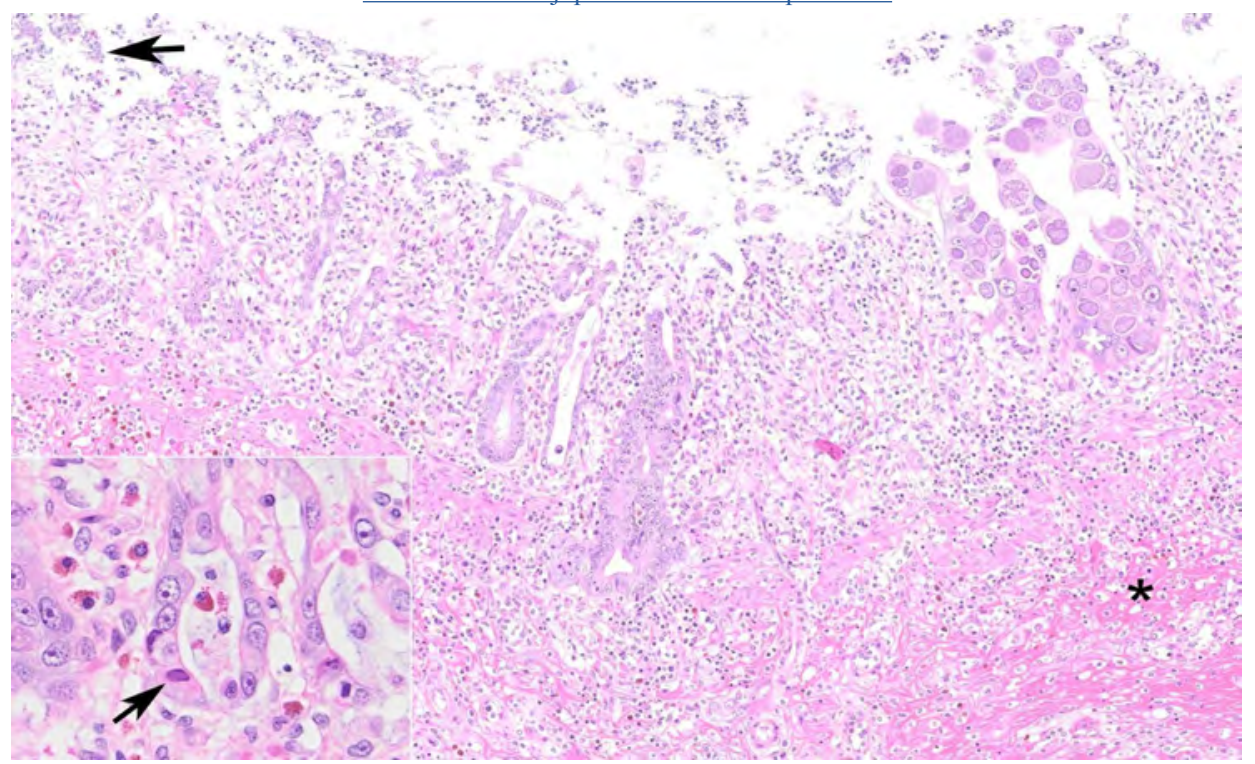

Figure 3. Small intestine, raccoon. Observe the collapse of the mucosa with superficial bacterial colonization (arrow) and intracellular coccidia (white asterisk). There is severe loss of crypts, diffuse lymphoplasmacytic, and neutrophilic infiltration. The submucosa is expanded by hemorrhage and cellular infiltration (black asterisk). Inset: Amphophilic intranuclear inclusion body (arrow) and hypertrophic enterocytes (regeneration) of the crypt epithelium. HE.

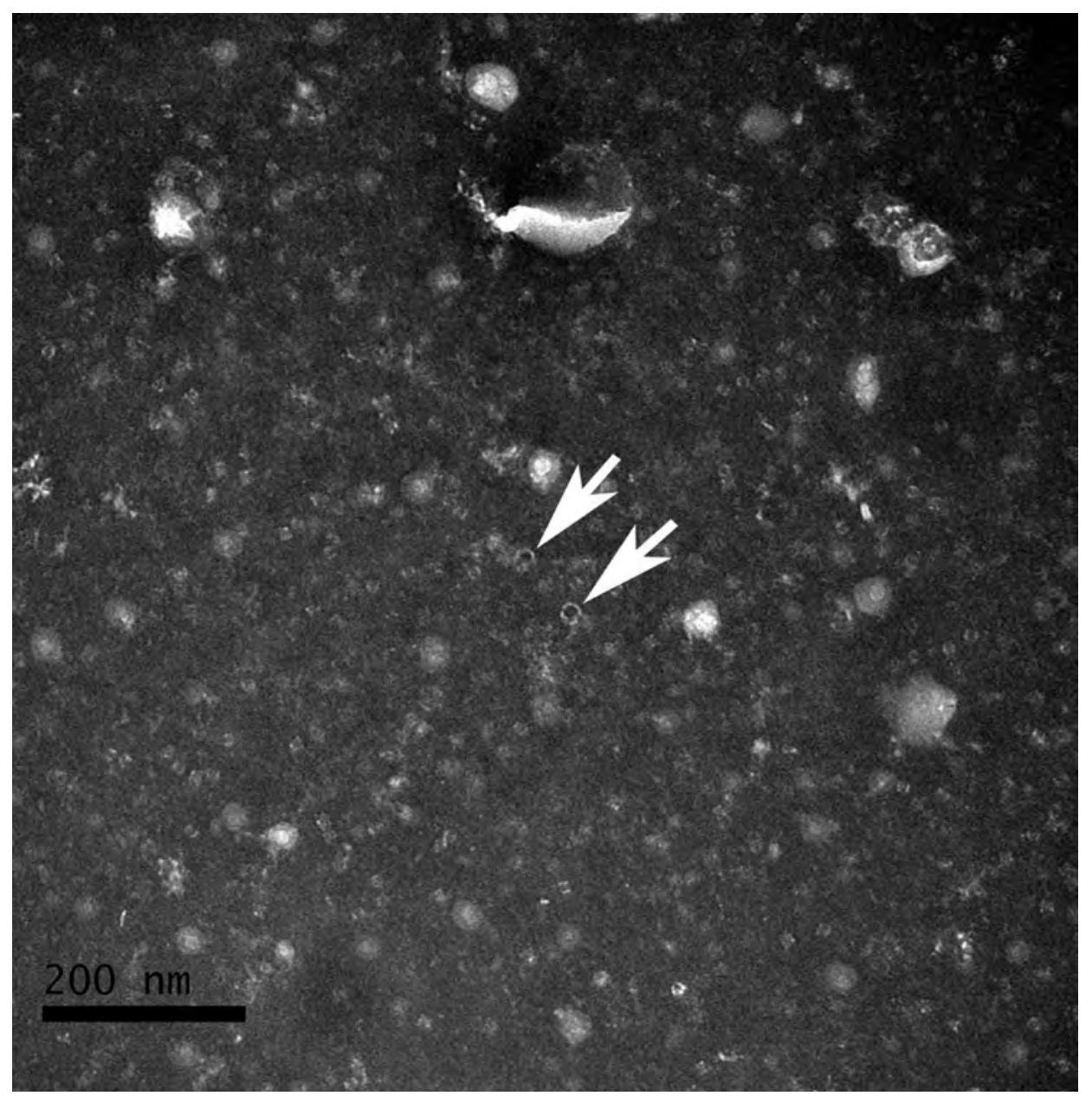

Figure 4. Negative staining of feces. Observe two empty virions with 20-22nm diameter, size is compatible with parvovirus family. Uranyl acetate. 


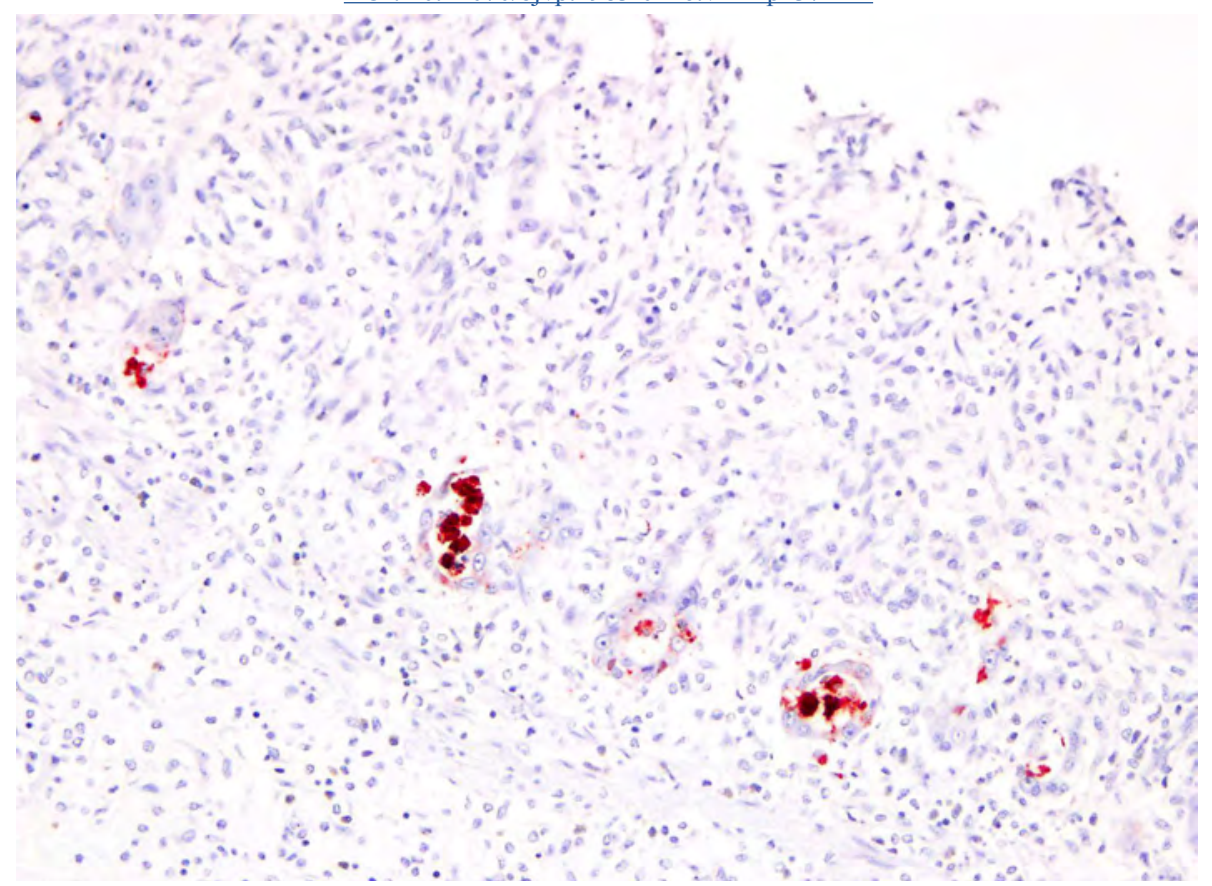

Figure 5. Small intestine, raccoon. Positive immunolabeling for parvovirus of the necrotic cells within the crypts. IHC.

\section{Typical microscopic findings of the disease:}

I. Severe collapse of the mucosa of the small intestine ('radiomimetic lesion');

II. Severe crypt epithelium necrosis with regeneration;

III. Rare amphophilic or basophilic intranuclear inclusion bodies ;

IV. Lymphoplasmacytic, necrotizing, hemorrhagic exudate;

V. Frequent opportunistic enteric infections like bacteria, protozoans (coccidia), fungi and/or nematodes.

\section{Etiological diagnosis:}

- Parvoviral enteritis

\section{Most common blood work abnormality: \\ - Lymphopenia}

\section{Discussion:}

Viral members of the family Parvoviridae such as feline parvovirus (FPV), canine parvovirus (CPV) and mink enteritis virus might occur in raccoons (Allisson et al, 2013; Church et al, 2018). From all these viruses, infection by CPVlike virus represents around $85 \%$ of all the parvovirus-associated infections in raccoons (Allison et al, 2013). CPV-2 has been isolated in North American raccoons (Allison et al, 2012; Kapil et al, 2012), which alerts its possible role in the dissemination of CPV-2 to companion animals (Kapil et al, 2012)

Thorough description of the clinical and pathological features of parvovirus infection in raccoons has not been carried out. Anecdotally, the disease tends to be more severe or fatal in juveniles than adults such as occurs in domestic animals (Church et al, 2018). The infected raccoons manifest lethargy, fever, diarrhea and vomiting (Kapil et al, 2010; Allison et al, 2012). The pathological findings observed in the studied raccoon are similar to those briefly described in a few reports and they include mucoid, watery and yellowish intestinal content with yellow material coating the smooth and glassy mucosa (Kapil et al, 2010). Histologically, lymphoid depletion is commonly observed, but the main lesions are localized in the small intestine, which has widespread crypt necrosis, sloughing of the villi, collapse of the lamina propria, regenerating crypt epithelial cells, fibrin, hemorrhage and bacteria coating the remaining mucosa (Kapil et al, 2010, Church et al, 2018). The intestinal infection by gram-negative bacteria elicits septicemia and endotexmia, which is common in fatal cases (Uzal et al, 2016). Positive immunolabeling in the affected intestine seems to be dependent of the remaining enterocytes holding viral antigens (Kapil et al, 2010). Thus, microscopic appearance along with the combination of IHC, electron microscopy and/or molecular testing would be necessary for a thorough diagnosis. In the studied raccoon, IHC and direct electron microscopy were both positive for parvovirus complementing the histopathological features. Common clinical finding among susceptible animals consists of lymphoid depletion (lymphopenia), lymphoid necrosis and necrohemorrhagic enteritis (Macartney et al, 1984; Church et al, 2018). Extraintestinal lesions generated by canine parvovirus have been recently reported in two juvenile raccoons with cerebellar hypoplasia and dysplasia (Wünshmann et al, 2020a), and encephalitis (Wünshmann et al, 2020b). 


\section{References}

1. Allison AB, Harbison CE, Pagan I, Stucker KM, Kaelber JT, Brown JD, Ruder MG, Keel MK, Dubovi EJ, Holmes EC, Parrish CR. 2012. Role of multiple hosts in the cross-species transmission and emergence of a pandemic parvovirus. J Virol 86: 865-872.

2. Allison AB, Kohler DJ, Fox KA, Brown JD, Gerhold RW, Shearn-Bochsler VI, Dubovi EJ, Parrish CR, Holmes EC. 2013. Frequent cross-species transmission of parvoviruses among diverse carnivore hosts. J Virol 87: 2342-2347.

3. Church ME, Terio KA, Keel MK. 2018. Chapter 12-Procyonidae, Viverridae, Hyenidae, Herpestidae, Eupleridae, and Prionodontidae, p. 305-316. In: Terio KA, McAloose D, St. Ledger J (Eds) Pathology of Wildlife and Zoo Animals. 1st ed. ELSEVIER, San Diego, USA.

4. Kapil S., Rezabek G., Germany B. Johnston L. 2010. Isolation of a virus related to canine parvovirus type 2 from a raccoon (Procyon lotor). Vet Rec 166: 24-25.

5. Macartney L, McCandlish IA, Thompson H, Cornwell HJ. 1984. Canine parvovirus enteritis 1: Clinical, haemotological and pathological features of experimental infection. Vet Rec 115: 201-210.

6. Uzal FA, Plattner BL, Hostetter JM. 2016. Alimentary system, p. 1-257. In: Maxie MG (Ed) Jubb, Kennedy, and Palmer's Pathology of Domestic Animals. 6th ed. ELSEVIER, Missouri, USA.

7. Wünschmann A, Lopez-Astacio R, Armién AG, Parrish CL. 2020a. Cerebellar hypoplasia and dysplasia in a juvenile raccoon with parvoviral infection. J Vet Diagn Invest 32:463-466.

8. Wünschmann A, Lopez-Astacio R, Armién AG, Reed L, Parrish CL. 2020b. Parvovirus-induced encephalitis in a juvenile raccoon. J Vet Diagn Invest, In Press. 